\title{
EXTRUSIÓN ORTODÓNCICA CON FIBROTOMÍA SUPRACRESTAL: CASO CON FIN PROTÉSICO
}

\author{
${ }^{1}$ Daniela Botero González, ${ }^{2}$ César Augusto Moncada Díez \\ ${ }^{1}$ Odontóloga U.CES, Medellín, (Colombia) \\ ${ }^{2}$ Ortodoncista U. CES, Docente F. Odontología U. CES, Medellín (Colombia)
}

Autor responsable de correspondencia: Daniela Botero González

Correo electrónico: danibgo@hotmail.com

\begin{abstract}
RESUMEN
La extrusión ortodóncica es el movimiento coronal de un diente por medio de la aplicación de fuerzas ortodóncicas. Este movimiento, acompañado de la fibrotomía supracrestal logra la migración del diente sin acompañamiento del periodonto. En este artículo se presenta el caso de un resto radicular de un incisivo central superior izquierdo, en paciente de sexo femenino de 40 años de edad, a la cual se le propone como tratamiento ideal para rehabilitar el diente, la realización de una extrusión ortodóncica acompañada de fibrotomía supracrestal. Se tienen en cuenta las consideraciones tanto del componente periodontal como del protésico, para este procedimiento. Se pretende, entonces, mostrar con este reporte de caso clínico, a la extrusión ortodóncica con fibrotomía supracrestal, como un método alternativo para el manejo de restos radiculares sin posibilidad de ser restaurados. Se logra exponer los bordes sanos y así obtener resultados protésicos adecuados en forma, función y estética. [Botero D, Moncada CA. Extrusión ortodóncica con fibrotomía supracrestal: caso con fin protésico. Ustasalud 2011; 10: 127 - 131]
\end{abstract}

Palabras clave: Extrusión ortodóncica. Movimiento dentario. Restauración dental. Periodonto

\section{ORTHODONTIC EXTRUSION WITH SUPRACRESTAL FIBEROTOMY: A CASE WITH PROSTHETIC END}

\begin{abstract}
Orthodontic extrusion is the crown movement of a tooth by orthodontic force application. This movement, accompanied by supracrestal fiberotomy accomplishes the migration of the tooth without the periodontium. This article reports the case of a residual root of an upper left central incisor in a female patient of 40 years old, to which is proposed as an ideal treatment to restore the tooth, making an orthodontic extrusion accompanied with a supracrestal fiberotomy. It is taking into the considerations of both the periodontal and the prosthetic component, for this procedure. The aim is, to show with this case report, the orthodontic extrusion with supracrestal fiberotomy, as an alternative method for management of root fragments without the possibility of being restored. Achieve to expose the healthy edges, and get appropriate prosthetic results in form, function and esthetics.
\end{abstract}

Key words: Orthodontic extrusion, Tooth movement, Dental restoration, Periodontium

Recibido para pubicación: 26 de septiembre de 2011. Aceptado para publicación: 10 de diciembre de 2011

\section{INTRODUCCIÓN}

La extrusión ortodóncica o también llamada erupción forzada, consiste en un movimiento vertical o coronal de la raíz, producido por la aplicación de fuerzas ortodóncicas. Todo esto, con la finalidad de ganar corona clínica. ${ }^{1-3}$

Las fuerzas ortodóncicas generan la expansión o distensión de las fibras periodontales y gingivales que arrastran todo el periodonto con el movimiento ortodóncico y se obtiene una migración coronal de la cresta alveolar y de los márgenes gingivales. ${ }^{1,2}$

Este procedimiento puede o no ser acompañado de una alternativa de tratamiento que sugirió Pontoriero en 1987, llamada fibrotomía supracrestal o re- sección de fibras. Esta consiste en hacer incisiones intracreviculares a través del epitelio de unión y de la inserción del tejido conectivo supracrestal. ${ }^{1,2,4,5}$ Con este procedimiento, se impide que el periodonto migre con la erupción del diente, debido a la eliminación en la tensión sobre el hueso de las fibras supracrestales, ${ }^{1,2,4,5}$ que son: las circulares, dentogingivales, dentoperiósticas y las transeptales. ${ }^{6,7}$

La fibrotomía supracrestal debe ser realizada idealmente cada 15 días, para obtener un aumento en la exposición radicular sin variar los niveles de la cresta alveolar ni de los márgenes gingivales, para eludir la necesidad de hacer una cirugía ósea correctiva al finalizar el tratamiento. ${ }^{1,2,4,8}$ La fibrotomía supracrestal se encuentra indicada en los casos, en el 
que el diente que se va a extruir, presenta alguna de las siguientes condiciones: perforación radicular supra o subgingival, nivelar y alinear el margen gingival cuando hay desarmonía estética, corregir fracturas radiculares verticales y horizontales en el tercio cervical o medio y resolver problemas de caries subgingivales. ${ }^{1}$

\section{REPORTE DE CASO}

Paciente de 40 años de edad, de sexo femenino, con estado de salud general bueno quien ingresa a tratamiento protésico con pregrado, a la clínica de la universidad CES, en Sabaneta. La paciente consulta con resto radicular del 21 con tratamiento endodóntico previo y provisional acrílico de termocurado, al retirar este último, se diagnostica caries intraconducto activa que compromete el tercio coronal de la raíz, en sus paredes vestibular y mesial. Se decide retirar la caries con cucharilla, con lo cual queda un socavado subgingival, de aproximadamente $3 \mathrm{~mm}$ (Figuras 1 y 2 ).

Como este diente va a ser rehabilitado protésicamente con un perno metálico y prótesis parcial fija, se decide realizar el procedimiento de extrusión ortodóncica con fibrotomía, por lo cual se remite a la paciente a la clínica de ortodoncia de pregrado. La extrusión ortodóncica debía ser realizada hasta que el socavado se encontrara a $2 \mathrm{~mm}$ supragingival, por orden del rehabilitador oral encargado. Al examen clínico de la arcada superior se encuentran provisionales acrílicos bien adaptados en 12, 11, 21 y 23, los dientes restantes se encuentran sanos y/o con restauraciones adaptadas.

En la clínica de ortodoncia de pregrado, de la universidad CES, se da inicio al tratamiento ortodóncico. Para esto, se utiliza un sistema con bracket estándar posicionado de 13 a 23. Se pone un arco de alambre TMA 0,017" x 0,025", realizado un ansa en caja para el 21 y se liga individualmente con elásticos. El bracket en el diente 21, con provisional acrílico, se ubicó de tal manera que quedara pasivo (Figura 3).

La fibrotomía supracrestal es realizada cada 15 días, previa anestesia local de la paciente, y se realiza con un mango de bisturí con hoja \# 15 y el alisado radicular cerrado se realiza con curetas de Gracey 3/4 y $7 / 8$ (Figura 4).

Luego de cuatro meses de tratamiento y la realización de seis fibrotomías supracrestales, el diente logra ser extruido $4 \mathrm{~mm}$ por encima del margen gingival, deja el margen más apical del socavado de la raíz a $2 \mathrm{~mm}$ por encima del margen gingival (Figuras 5 a 7) como fue ordenado por el rehabilitador oral, para ser correctamente tallado posterior a la fase de retención ortodóncica, que se realizó por 3 meses.
(Figura 8). Para esta fase, el bracket fue reposicionado en el provisional acrílico de termocurado y se utilizó un arco de alambre acero 0,016" x 0,016" y se liga individualmente con elásticos.

Se procede luego de tener el resultado final de la extrusión ortodóncica con fibrotomía supracrestal, a realizar el tallado y desobturación endodóntica del resto radicular (Figura 9), queda así, el diente preparado para la realización de su tratamiento endodóntico y protésico; con su respectivo perno y prótesis parcial fija.

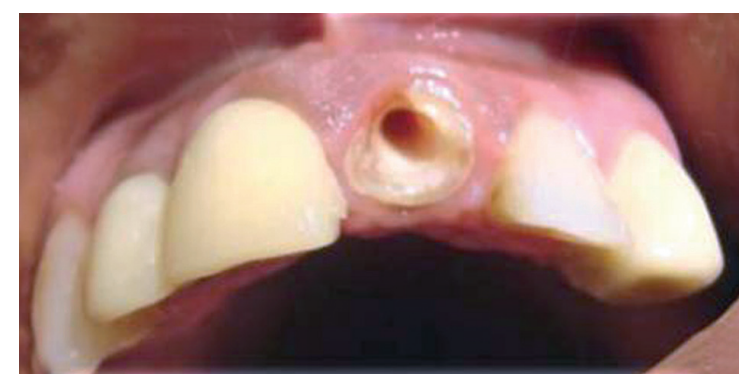

Figura 1. Resto radicular del 21, sin caries dental y con el socavado en vestibular y mesial

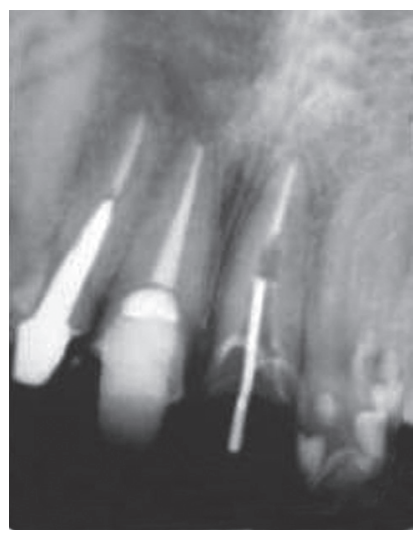

Figura 2. Radiografía periapical del estado inicial del diente
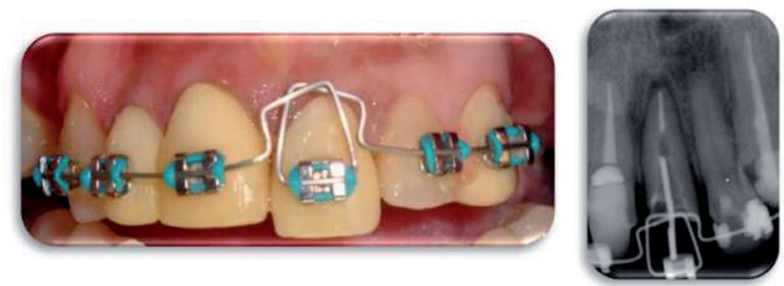

Figura 3. Fotografia y radiografia periapical del seguimiento 1
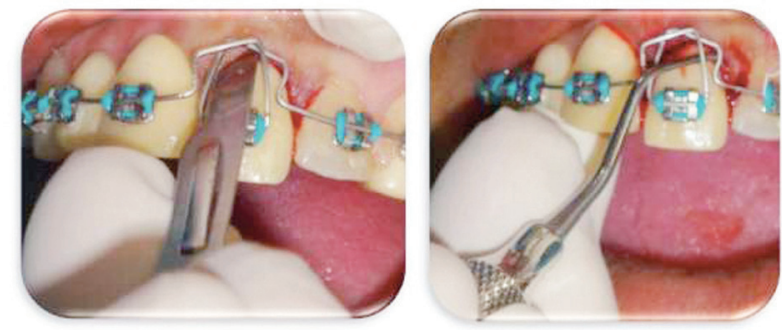

Figura 4. Fibrotomía supracrestal 


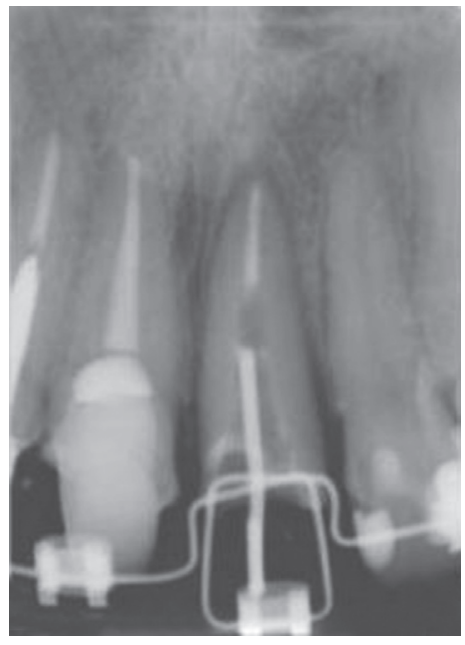

Figura 5. Radiografia periapical del seguimiento 2

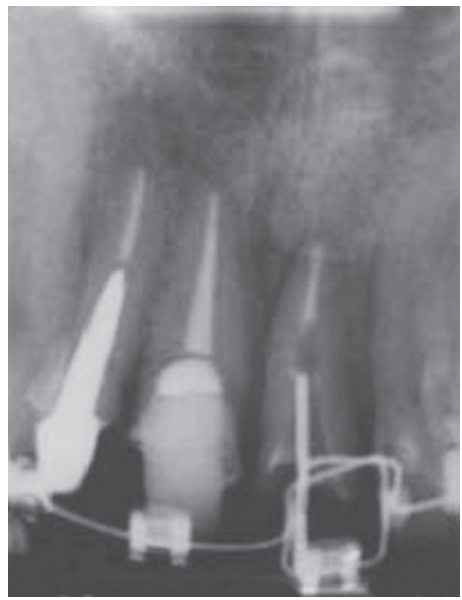

Figura 6. Radiografia periapical del seguimiento 3

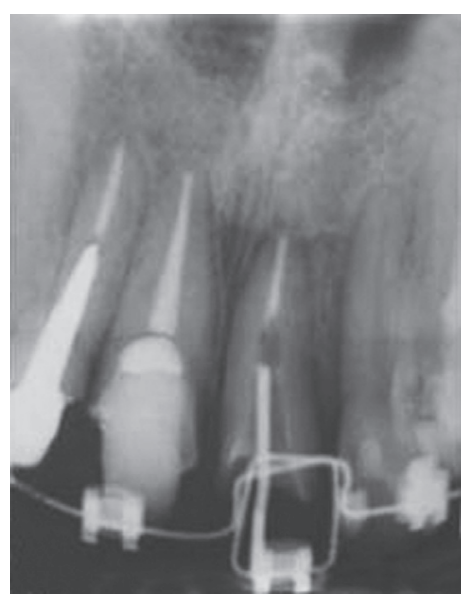

Figura 7. Radiografía periapical del seguimiento 4
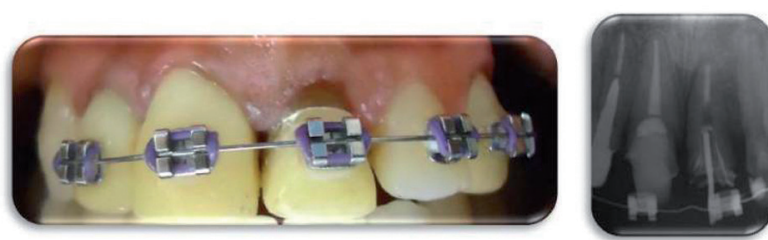

Figura 8. Fotografia y radiografia periapical de la fase de retencion

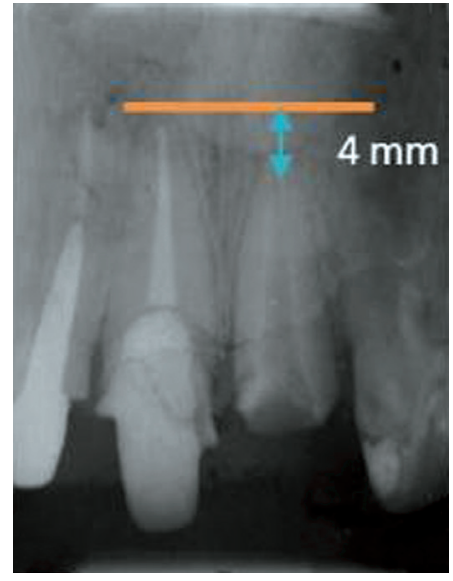

Figura 9. Radiografia periapical del resultado final, con el resto radicular tallado y desobturado

\section{Consideraciones del tratamiento}

Los pacientes indicados para realizar este procedimiento son aquellos que han sufrido fracturas radiculares a nivel de la cresta ósea, dientes con reabsorción radicular interna o externa, perforaciones iatrogénicas, caries infraóseas, dientes con defectos periodontales de 1 o 2 paredes, para mejorar el contorno gingival con los dientes adyacentes y para manejar defectos óseos. ${ }^{5,8,9}$

Así mismo, se debe tener presente durante el procedimiento, ciertas consideraciones para evitar alguna complicación, estas son: aplicación controlada de las fuerzas, vitalidad del ligamento periodontal, adecuado anclaje, reconocer si la forma de la raíz es favorable para ser extruida y controlar los problemas pulpares si el diente es vital. ${ }^{8}$

Respecto al sistema ortodóncico para realizar un tratamiento como la extrusión ortodóncica con fibrotomía supracrestal y habida cuenta de su fin protésico, se deben considerar al momento del posicionamiento de los brackets, lo que se mencionará a continuación.

El sistema ortodóncico, debe ser rígido con los dientes anclas y muy flexible con el diente que se va a extruir. Se logra así, que en una semana el diente se extruya menos de $1 \mathrm{~mm}$. ${ }^{1,10}$ La fuerza de extrusión máxima que se debe aplicar para crear un movimiento extrusivo lento, es de $30 \mathrm{~g}$ aproximadamente. ${ }^{5,10,11}$ En la literatura se recomienda fuerzas de $15 \mathrm{~g}$ para las raíces delgadas de los incisivos inferiores y de $60 \mathrm{~g}$ para las molares. ${ }^{10,11} \mathrm{Y}$ esta fuerza puede realizarse sobre una restauración en resina y/o acrílico. ${ }^{1,11}$

Es muy importante estabilizar el diente extruido por medio de un periodo de retención adecuado, para prevenir la intrusión por recidiva. ${ }^{1,8,11,12} \mathrm{El}$ reordenamiento de las fibras del ligamento periodontal 
que están unidas al hueso se completa en 28 días, aproximadamente. ${ }^{1,11,12}$

\section{Cambios en el periodonto con la extrusión ortodóncica}

La extrusión de los dientes por la aplicación de fuerzas ortodóncicas tiene implicaciones directas sobre el periodonto. ${ }^{1,5,13}$ Esto se basa, en que al producirse el movimiento de extrusión del diente también ocurre simultáneamente el desplazamiento coronario del aparato de soporte y el tejido gingival. ${ }^{13,14}$

A medida que se extruye el diente la raíz se mueve en dirección coronal en el alvéolo y se estiran las fibras gingivales y del ligamento periodontal para seguir al diente en su movimiento. ${ }^{1,5,15}$ Esto, se proporciona gracias a que el tejido gingival es fijado a la raíz por el tejido conectivo. ${ }^{1}$

Los cambios en el ligamento periodontal se dan en las fibras principales del mismo y adquieren un desplazamiento en dirección coronal de las fibras, que asumen una posición más horizontal. ${ }^{1,8}$ En el tejido gingival se presenta un incremento en la amplitud de la encía adherida y en la unión mucogingival el movimiento coronal es relativamente constante. ${ }^{1,8,10,13}$

La cantidad de encía adherida es aumentada a través de la eversión del epitelio surcular, aparece primero como un tejido no queratinizado inmaduro y luego como un tejido queratinizado. Este proceso de queratinización requiere de 28 a 42 días. Luego que ocurre el movimiento coronal del tejido periodontal se requiere una mínima corrección quirúrgica periodontal, para evitar o minimizar esta corrección, se recomienda la fibrotomía supracrestal. ${ }^{10}$

La aplicación de una fuerza continua, resulta en un movimiento tardío del diente en dirección vertical, con aposición ósea tanto en el fondo del alvéolo, como en la cresta alveolar. ${ }^{1,5,8}$ Como se evidencia en las radiografías periapicales del caso clínico.

\section{Consideraciones protésicas de la extrusión ortodóncica}

La restauración protésica posterior a una extrusión dental, posibilitan la obtención de un resultado biológico, estético y funcionalmente más predecible. ${ }^{13}$

Son numerosas las causas, por las que un diente puede presentar destrucción coronal extensa o incluso llegar a perder la corona. Al restaurar un diente con alguna de estas dos características, el objetivo principal es el de lograr una adecuada cantidad de estructura dentaria sana para colocar el margen de una restauración. ${ }^{5,16}$

Lo anterior se realiza, debido a que al conservar el diente en boca por unos años más, se logró conser- var el espacio biológico, una correcta proporción corona-raíz, se preserva la integridad del reborde alveolar en altura y anchura, la encía sana y el mantenimiento del espacio para la restauración protésica futura. ${ }^{5,16}$

Las indicaciones protésicas para realizar una extrusión ortodóncica, son: optimización de la proporción corona-raíz de los dientes pilares, optimización de la ubicación intra-arco e inter-arco de dientes previo a la restauración definitiva, nivelación del plano incisal, nivelación de los márgenes gingivales y el establecimiento de una guía anterior adecuada para proteger las restauraciones del segmento anterior. ${ }^{1}$

Es así, como los dientes o restos radiculares con un pronóstico reservado, logran mediante la extrusión ortodóncica ser aptos para una rehabilitación protésica adecuada, de lo contrario serían extraídos. ${ }^{17}$

\section{DISCUSIÓN}

Existen diversas causas por las cuales un diente, puede perder la corona clínica más allá del margen gingival o incluso por debajo de la cresta ósea, a tal punto, de no poder ser restaurado por los métodos convencionales sin comprometer la retención, durabilidad y estética de la restauración. ${ }^{18}$

La técnica de extrusión ortodóncica es, en muchos casos, el método alternativo para el manejo de restos radiculares o dientes aislados, sin posibilidad de ser restaurados, se logra con esta técnica, exponer los bordes sanos, para luego lograr resultados protésicos adecuados en forma, función y estética, sin alterar la armonía de los dientes vecinos. ${ }^{18}$

Estudios que realizan una evaluación métrica del movimiento gingival asociado a la extrusión ortodóncica de los incisivos indicaron que la encía libre y la encía adherida se movieron aproximadamente en el $80-90 \%$ de la distancia total extruida, incrementándose la corona clínica y el ancho total de la encía. ${ }^{15}$

Debido a esto y para evitar la intrusión del resto radicular después de la extrusión ortodóncica, se realizó la fibrotomía periodontal supracrestal estirada. Para que cuando estas se reorganizaran en la nueva posición del resto radicular, permitieran volver a establecer el espacio biológico. ${ }^{19,20}$

Además, la extrusión ortodóncica se considera un procedimiento seguro con respecto a la aparición de reabsorción ósea y/o radicular, ya que como el movimiento es vertical, la raíz no se desplaza a través del hueso, ni comprime el ligamento periodontal. Tampoco implica la pérdida de soporte periodontal o tejido óseo de los dientes vecinos, lo que favorece la estética. ${ }^{5,19}$ Es por esto que la extrusión ortodón- 
cica con fibrotomía supracrestal, fue el tratamiento de elección para este caso clínico en particular.

\section{Conclusiones}

El estado actual de los conocimientos sobre el tratamiento de los dientes traumatizados, se basa principalmente en los casos clínicos. ${ }^{16}$

La extrusión ortodóncica como procedimiento para salvar dientes y en especial los incisivos centrales, ha sido recomendada por autores como Goldson y Malmgren (1981), Kahnberg (1985) y Caliskan (1998). Sus estudios muestran cómo los dientes con fracturas que comprometen la raíz, logran con la extrusión sobrepasar la inserción epitelial para ser preparados con líneas de terminación protésicamente adecuadas. ${ }^{12,16,21}$

La extrusión ortodóncica con fibrotomía supracrestal es considerada una excelente técnica y muy beneficiosa al generar movimientos dentales controlados, tanto para los odontólogos que se comprometen en la rehabilitación dental, como para el paciente que desea mantener sus dientes., ${ }^{9,16}$

\section{BIBLIOGRAFÍA}

1. Uribe GA, Cadavid GJ, Jaramillo A, Mejía A. Extrusión ortodóncica o erupción forzada. En: Uribe GA. Ortodoncia: teórica y clínica. 2da. ed. Medellín: Corporación para Investigaciones Biológicas; 2010. Pp. 1064-1085

2. Chávez L, Walter A, Puigdollers A. Extrusión ortodóncica. Rev Oper Dent Endod. 2006; 5: 47

3. Valverde HR, Balarezo JA, Urquiaga CL, Revoredo A. Extrusión ortodóncica, una alternativa simple a un problema protésico. Rev Estomatol Herediana. 2000; 10: 37-40

4. Heda CB, Heda AA, Kulkarni SS. A multi-disciplinary approach in the management of a traumatized tooth with complicated crown-root fracture: A case report. J Indian Soc Pedod Prev Dent. 2006; 24: 197 - 200

5. Rey D, Johnson N. Extrusión ortodóncica: Una alternativa para la regeneración periodontal y la restauración. CES Odontología. 1998; 11: 54 - 58

6. Lindhe J, Karring T, Araújo M. Anatomy of the periodontium. In: Lindhe J, Karring T, Lang NP. Clinical periodontology and implant dentistry. $4^{\text {th }}$. ed. Copenhagen: Blackwell Munksgaard; 2003. Pp. 3 - 49

7. Itoiz ME, Carranza FA. The gingiva. In: Newman MG, Takei HH, Carranza FA. Clinical periodontology. $9^{\text {th }}$. ed. Philadelphia: W.B. Saunders Company; 2002. Pp. 16 - 35

8. Barrera L. El periodonto ante los movimientos de extrusión e intrusión ortodóntica. CES Odontología. 2005; 18: $47-58$

9. Bach N, Bayland JF, Voyer R. Orthodontic extrusion: Periodontal consideration and applications. J Cant Dent Assoc. 2004; 70: 775 - 780

10. Dannan M, Darwish M, Sawan M. The Orthodontic Extrusion Movements and the Periodontal Tissues. The Internet Journal of Dental Science. 2009; 8 (1).

11. Brindis MA, Block MS. Orthodontic tooth extrusion to enhance soft tissue implant esthetics. J Oral Maxillofac Surg. 2009; 67: $49-59$
12. Koyuturk AE, Malkoc S. Orthodontic extrusion of subgingivally fractured incisor before restoration. A case report: 3-years follow-up. Dent Traumatol. 2005; 21: $174-178$

13. Santon D, Asenjo M, Passanezi E. Extrusión ortodóntica para la reconstrucción de la papila interdental perdida en incisivos centrales superiores restaurados con coronas de cerámica - Reporte de caso clínico. Rev Odontol Dominic. 2002; 8: $7-15$

14. Sönmez H, Tunc ES, Dalcı ON, Saroglu I. Orthodontic extrusion of a traumatically intruded permanent incisor: A case report with a 5-year follow up. Dental Traumatol. 2008; 24: $691-694$

15. López C. Orthodontics-Periodontics, Part I. Review article. Rev Chil Periodon Oseoint. 2004; 1: 12 - 19

16. Medeiros RB, Mucha JN. Inmediate vs. late orthodontic extrusion of traumatically intruded teeth. Dental Traumatol. 2009; 25: $380-385$

17. Pascual A, Nieves C, González E, Martínez M, Soca L. Extrusión dentaria en el tratamiento de una fractura mixta. Medisan. 2008; 12 (2)

18. D’Ercole V, Rodríguez F. Erupción forzada de dientes siguiendo la técnica seleccional de doble arco con fines protésicos. Acta Odontol Venez. 2003; 41: 140 - 143

19. Hamata C, Heidy M, Lemos J, Koogi C, Rangel I, Magro G, Regina S. Management of a complicated crown-root fracture using adhesive fragment reattachmentand orthodontic extrusion. Dent Traumatol. 2009; 25: 541 -544

20. Bondemark L, Kurol J, Hallonsten A, Andreasen JO. Attractive magnets for orthodontic extrusion of crown-root fractured teeth. Am J Orthod Dentofac Orthop. 1997; 112: $187-193$

21. Malmgren O, Malmgren B, Frykholm A. Rapid orthodontic extrusion of crown root and cervical root fractured teeth. Endod Dent Traumatol. 1991; 7: 49 - 54
Correos electrónicos de los autores:

Daniela Botero González: danibgo@hotmail.com César Augusto Moncada Díez: cmoncada@odontosur.com.co 